Research Article

\title{
Kinematic Analysis of a Novel System for Paper Rewinding Machines
}

\author{
Gianluca Gatti $\mathbb{D}^{1},{ }^{1}$ Lorenzo Lupi, $^{2}$ and Giuseppe Lupi ${ }^{2}$ \\ ${ }^{1}$ Department of Mechanical, Energy and Management Engineering, University of Calabria, Rende 87036, Italy \\ ${ }^{2}$ United Converting Tissue Srl, Lucca 55025, Italy \\ Correspondence should be addressed to Gianluca Gatti; gianluca.gatti@unical.it
}

Received 1 February 2021; Revised 6 April 2021; Accepted 16 April 2021; Published 6 May 2021

Academic Editor: Emiliano Mucchi

Copyright ( 2021 Gianluca Gatti et al. This is an open access article distributed under the Creative Commons Attribution License, which permits unrestricted use, distribution, and reproduction in any medium, provided the original work is properly cited.

Rewinding machines are used in the paper converting industrial sector to unroll paper veils from large source reels and wind them around smaller rolls, for the production of paper logs to be used in household activities. Many different types of rewinding machine exist, and they are mostly based on stapling devices which automatically unroll a large coil of paper, roll it up around a new log, stop the veil allowing it to be torn off, unload the completed paper log, and reload a new paper core to be rolled up. Winding units generally include a primary and a secondary roller, supplying the desired rotational speed for winding, and a pressure unit consisting of a pressure roller in contact with the paper log being rolled up, which assures adequate containment and avoids the paper log to escape. All known systems have some mechanical limitations, mainly due to the contact of the pressure roller with the paper log, causing instabilities and vibrations, particularly when working with soft paper veils. This limits the maximum possible winding speed. The aim of this paper is it to present a detailed kinematic analysis of a novel pressure unit which overcomes the aforementioned limitation.

\section{Introduction}

Rewinding machines are used in the paper converting industrial sector to unroll large reels of paper veils and wind them around smaller logs for the commercial sector, e.g., paper rolls for household keeping. In its most common configuration, a rewinding machine comprises a drive unit which supplies the paper veil, a winding unit for winding the paper veil supplied by the drive unit around a winding core, a primary and a secondary winding roller rotating in contact with the core being rolled up, and a pressure unit equipped with a pressure roller in contact with the paper log. During the winding phase, the peripheral speed of the rollers is substantially equal to the winding speed of the veil.

In its classical configuration, the pressure unit consists of one single pressure roller which is accomplishing a circular reciprocating motion around a fixed axis of rotation [1]. In [2], a discussion is presented on the application and limitations of reels in paper winding machines used for finished rolls. However, the interest of the scientific community towards this topic has been mainly limited to the control system to feed the paper veil and assure adequate tension to avoid defects in the final product. In [3], a tension control system is proposed which realizes stable tension of the rewinding process overcoming the influence of external disturbances. In [4], a mechanical modification was introduced to improve the stability of a commercial rewinding machine. In [5], a control system for paper rewinding machine based on PLC is presented along with the control functions of unwinding tension and rewinding force. In [6], robust tension estimators were developed considering dynamic, friction, and inertial effects, to provide stable and precise sheet tension control without the use of load cells. In [7], the aim was to replace the signals from the load cell and the absolute encoder with their estimated values. In [8], a closed loop control system was proposed to overcome the effect of any disturbance during machine operations and improve the quality of the final product. In [9], a way of self-adjusting reel diameter calculation is presented providing superior reeling performance. In [10], a pressure-control scheme for a 
hydraulic actuator is proposed to improve the tightness of the paper reel, and in [11], the influence of the alignment of the rolling elements on the product quality is concisely discussed.

As far as the authors are aware, except the work presented in [1], no significant contribution has been addressed in the scientific literature to the proposal of novel mechanical systems to improve at source the rewinding process in the paper industry. This paper thus proposes the kinematic analysis of a new pressure unit [12] which is able to improve the stability of the paper log being rolled up and thus increase the winding speed of the whole process.

\section{Description of the System}

Figure 1 illustrates a model of the system under study and shows the main components of the mechanism for winding a paper log. This is formed by the rotation of two winding rollers, referred to as the upper and lower winding roller, respectively. The paper log is kept in contact with these two rollers by means of a pressure unit, which is the proposed novelty in the machine. It consists of a main oscillating arm, rotating around a fixed axis A, coupled to a secondary arm (i.e., the triangular-shaped plate in Figure 1) via a rotational joint along axis B. The secondary arm supports three pressure rollers whose purpose is to apply the appropriate pressure to the paper log being rolled up, while keeping it in contact to the winding rollers. The pressure unit is controlled by means of two actuators: one revolute motor in $\mathrm{B}$ and one revolute motor in $\mathrm{A}$ or alternatively by means of a linear actuator as shown in Figure 1.

The different steps related to the paper log being rolled up are illustrated in Figure 2, where a sequence of snapshots is presented to clarify the motion of the system. Animation So, provided in the Supplementary Material (Available here) for this paper, shows an animation of the proposed system and compares it to the traditional one. The different phases are characterized by the type of contact between the paper log being rolled up and the other rollers involved in the process, which assure the paper log stability during winding. The instability which may appear in a traditional pressure unit is pictorially emphasized in the animation S0, provided in the Supplementary Material (Available here). Figure 2(a) then shows the initial phase where the paper core is loaded and introduced between the winding rollers, and an initial paper veil is rolled up and glued around it. The paper log is thus pushed towards one of the pressure roller of the pressure unit, which holds its position ready for engagement. The critical issues during this phase are related to the geometric dimensions and constraints of the system, which may prevent the pressure rollers to get close enough to the $\log$ being rolled up without interfering with the other winding rollers. Figure 2(b) shows the case where the paper $\log$ has reached a certain diameter. In this case, the paper log is in contact with three rollers-two winding rollers and one pressure roller. To prevent the paper log to escape through the spaces between the rollers, the pressure roller must follow a prescribed trajectory. Such trajectory has to assure that the geometrical segment between the centres of rotation

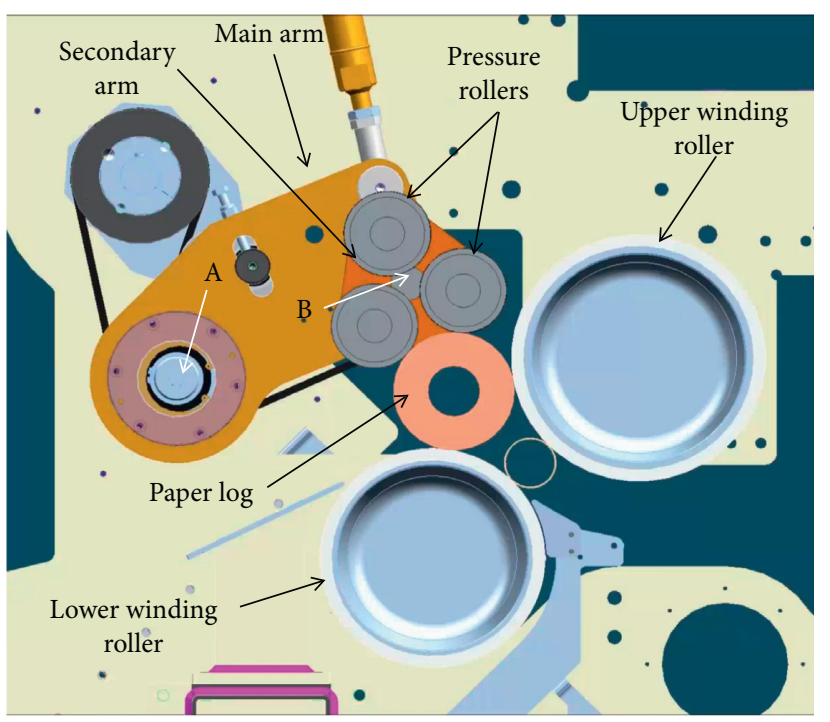

Figure 1: Computer-aided model of the main components in a paper rewinding machine.

of the two winding rollers is always perpendicular to the line passing through the paper log centre and the centre of rotation of the pressure roller being in contact with the paper $\log$. Figures 2(c) and 2(d) show two snapshots of the subsequent phases-the pressure unit rotates around axis $\mathrm{B}$ clockwise to assure the motion requirement discussed in the proceeding phase and convoy the paper log being rolled up. Such a rotation continues until a second pressure roller comes in contact with the paper log. This is a critical condition because it involves the transition from one specific trajectory to a different one. From this condition on, until the next phase, the paper log is constrained by four rollers (two pressure rollers and two winding rollers), which guarantee for the best condition of stability during the paper $\log$ winding. Figure $2(\mathrm{e})$ shows the situation where the paper $\log$ is about to be completed and it is led to ejection by loosing contact with the upper winding roller. The paper log is then in contact with three rollers, two pressure rollers and one winding roller. During this phase, as shown in Figure 2(b), the motion of the pressure unit has to assure an optimal trajectory to avoid paper log instability and escapement. Figure $2(\mathrm{f})$ shows the final phase where the paper winding has been completed and paper log is ejected. The pressure unit goes back to its initial position and is ready for the next product engagement, after a rotation of $120^{\circ}$.

In the following analysis, it is assumed that the system has nominal geometry, i.e., no geometrical or assembly error is considered. Well-established methods for kinematic calibration [13] can be adopted on the physical system for parameters identification. Since this paper focuses on the kinematics of the device, other issues related to rotor dynamics and defective ball bearing [14] are not considered. Also, contact issues between the paper log and the machine rollers as well as the related dynamics are not considered in this study. This is motivated by the focus of the paper and the fact that the stiffness of the paper log is negligible respect to that of the machine rollers. 


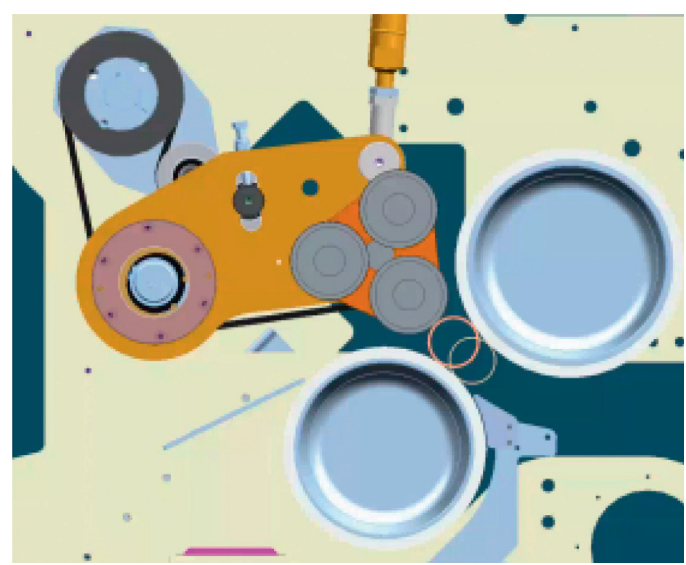

(a)

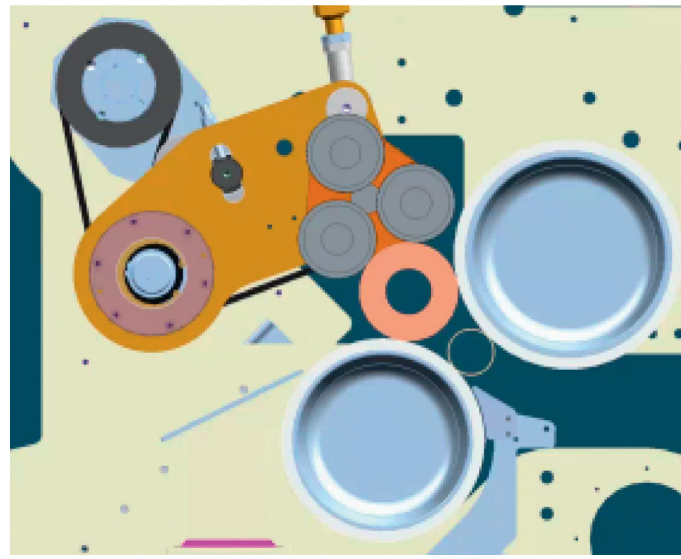

(c)

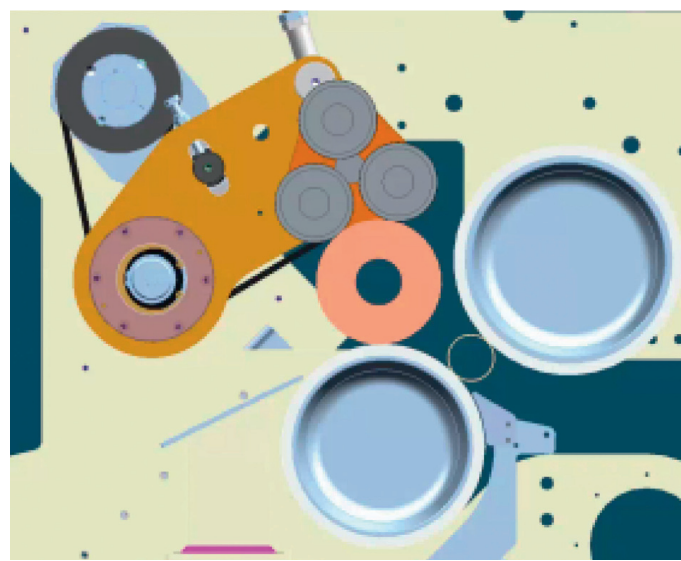

(e)

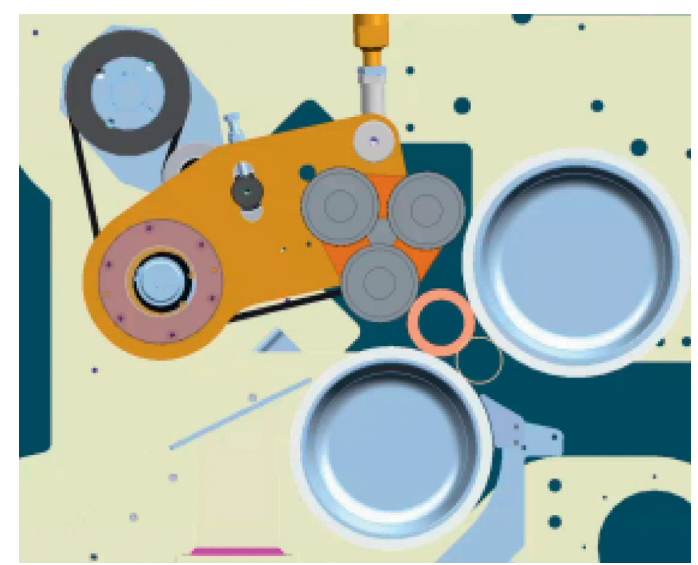

(b)

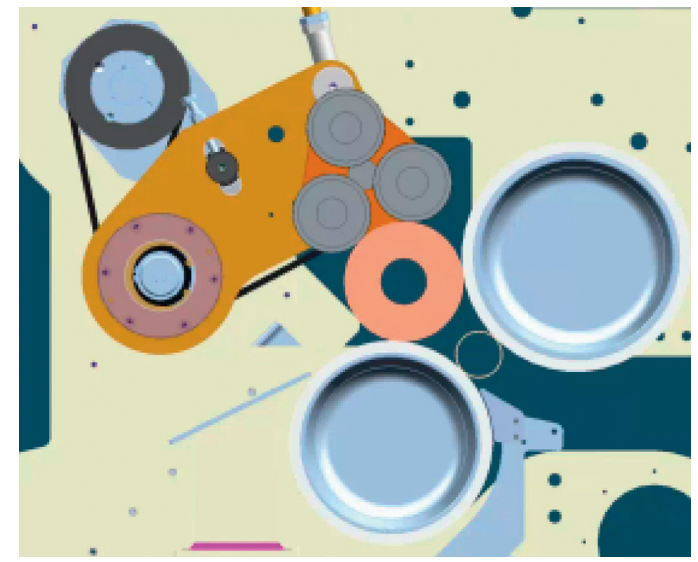

(d)

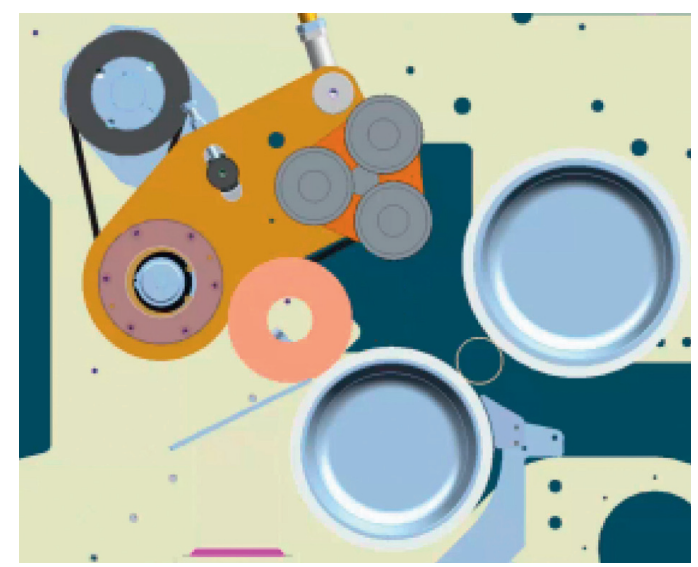

(f)

FIGURE 2: Snapshots of the system in motion: (a) initial phase involving the paper winding core, (b) paper log being rolled up in contact with one pressure roller, (c-d) paper log being rolled up in contact with two pressure rollers, (e) paper log being completed, and (f) paper log being ejected.

\section{Trajectory of the Paper Log}

From a kinematic point of view, the system in Figure 1 can be represented by the schematic drawing reported in Figure 3.

The aim of the present analysis is to determine the trajectory of the paper log being rolled up. This is then used in the next section to determine the trajectory of the pressure unit according to the requirements for stability, mentioned in the previous section.

From a kinematic point of view, the motion of the system and its component trajectories are expressed as a function of the increasing radius of the paper log being rolled up, $R_{L}$, which is a function of the paper length wrapped per unit time, i.e., the winding speed, $V$, according to the following relation: 


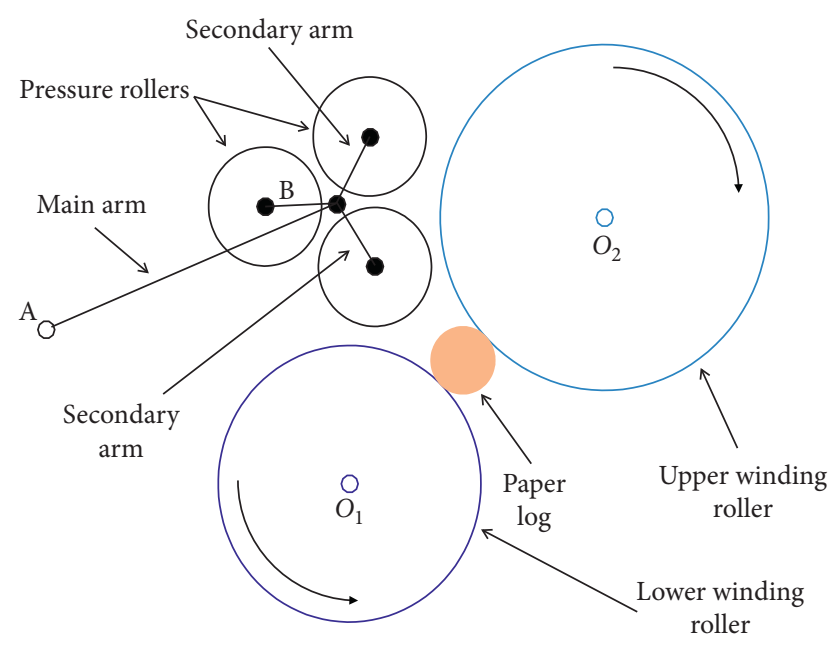

FIGURE 3: Schematic drawing of the system.

$$
R_{L}=\sqrt{R_{L i}^{2}+\frac{s V t}{\pi}},
$$

where $R_{L i}$ is the initial radius of the paper log, which coincides with the paper winding core, $t$ is the time, and $s$ is the theoretical paper thickness calculated as

$$
s=\frac{\pi\left(R_{L f}^{2}-R_{L i}^{2}\right)}{L},
$$

where $R_{L f}$ is the final radius of the completed paper $\log$ and $L$ is the paper wrapped on each paper log.

With reference to the snapshots in Figure 2, the characteristic phases occurring during the system motion are summarized in Figure 4.

During Phase I in Figure 4(a), the pressure unit is in starting configuration, with pressure roller [C] waiting for the paper $\log [\mathrm{F}]$ to initiate its winding. Such configuration is limited by the collision of the pressure rollers with the winding rollers [R1] and [R2].

During Phase II in Figure 4(b), the paper log reaches the engaging diameter, and the pressure unit begins to move, convoying the paper log being rolled up.

During Phase III in Figure 4(c), the paper log increases its diameter and the pressure unit moves and orients to assure an optimal trajectory. This can be defined as the one which guarantees that the line of pressure between the pressure roller [C] and the paper log [F] is perpendicular to the segment $\mathrm{O}_{1} \mathrm{O}_{2}$ in Figure 3. This can be cinematically achieved since the pressure unit is a two degrees-of-freedom mechanism.

During Phase IV in Figure 4(d), the pressure unit continues its motion, rotating both the main and secondary arms, until a second pressure roller [D] comes in contact with the paper log. This latter is then convoyed by two winding rollers and two pressure rollers until the final diameter is reached.

During Phase V in Figure 4(e), the paper log disengages from the upper winding roller, and it is kept in contact with the lower one thorough the motion of two pressure rollers [C] and [D] until ejection.

In the final Phase VI in Figure 4(f), the product is ejected, and the pressure unit returns back to its initial configuration waiting for the next engagement. The secondary arms rotate $120^{\circ}$, and the pressure roller [D] replaces the pressure roller [C] in Phase I.

During Phases I to IV in Figure 4, the paper log is always in contact with both winding rollers.

To perform a mathematical analysis, the following parameters are defined, according to Figure 5. $R_{1}\left(R_{2}\right)$ is the radius of the lower (upper) winding roller; $d$ is the constant length of the segment $O_{1} O_{2}$ between the rotational axes of the lower and upper winding rollers; $L_{1}\left(L_{2}\right)$ is the variable distance between the axis of rotation of the lower (upper) winding roller and the instantaneous axis of the rotation of the paper log, $F$; $\phi$ is the constant angle between the horizontal axis and the segment $O_{1} O_{2}$; and $\phi_{1}\left(\phi_{2}\right)$ is the variable angle between the horizontal axis and the segment $O_{1} F$ $\left(\mathrm{O}_{2} \mathrm{~F}\right)$. An orthogonal frame of reference $x y$ is also defined with origin at the axis of rotation of the lower winding roller, $\mathrm{O}_{1}$.

According to the system geometry, the paper log is initially being moved along the line perpendicular to the segment $\mathrm{O}_{1} \mathrm{O}_{2}$.

To ensure continuity of motion, it is required that the paper log is kept in contact with both winding rollers, from the initial diameter to the final diameter.

The following geometric constraints must thus be assured during motion:

$$
\begin{gathered}
L_{1} \cos \left(\phi_{1}\right)-L_{2} \cos \left(\phi_{2}\right)-d \cos (\phi)=0, \\
L_{1} \sin \left(\phi_{1}\right)-L_{2} \sin \left(\phi_{2}\right)-d \sin (\phi)=0,
\end{gathered}
$$

where $L_{1}=R_{1}+R_{L}, L_{2}=R_{2}+R_{L}$.

Squaring and adding equation (2), the following relation is obtained:

$$
L_{1}^{2}+L_{2}^{2}-2 L_{1} L_{2}\left[\cos \left(\phi_{1}\right) \cos \left(\phi_{2}\right)+\sin \left(\phi_{1}\right) \sin \left(\phi_{2}\right)\right]=d^{2},
$$

which can be conveniently rewritten using trigonometric formulas as

$$
\Delta \phi=\phi_{1}-\phi_{2}= \pm \arccos \frac{-d^{2}+L_{1}^{2}+L_{2}^{2}}{2 L_{1} L_{2}} .
$$

The ambiguity in the sign in equation (4) comes from the existence of two solutions to the problem-the one corresponding to the plus sign is where the paper log in Figure 5 moves along the positive $x$ direction and the one with the minus sign is where the paper log in Figure 5 moves along the negative $x$ direction. This latter is the one of interest in the specific problem under investigation.

Substituting equation (4) into equation (2), after some trigonometric manipulation, the following linear system of equations is obtained in the unknowns $\cos \left(\phi_{1}\right)$ and $\sin \left(\phi_{1}\right)$ : 


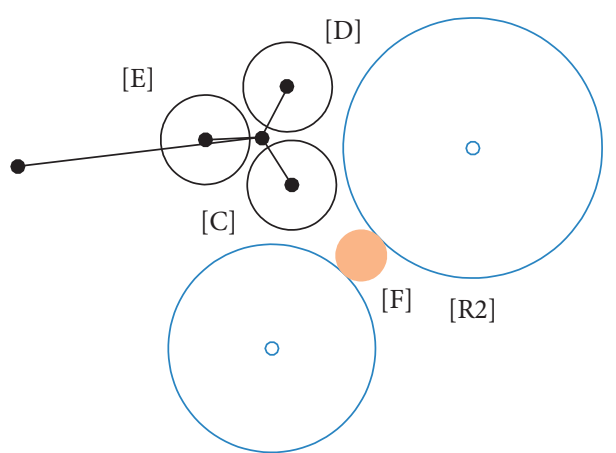

[R1]

(a)

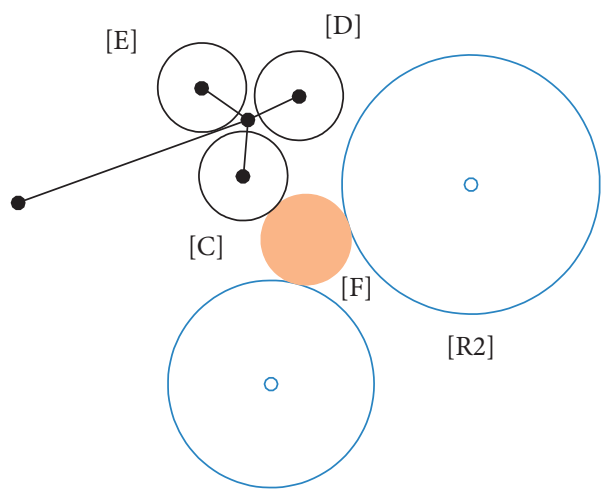

[R1]

(c)

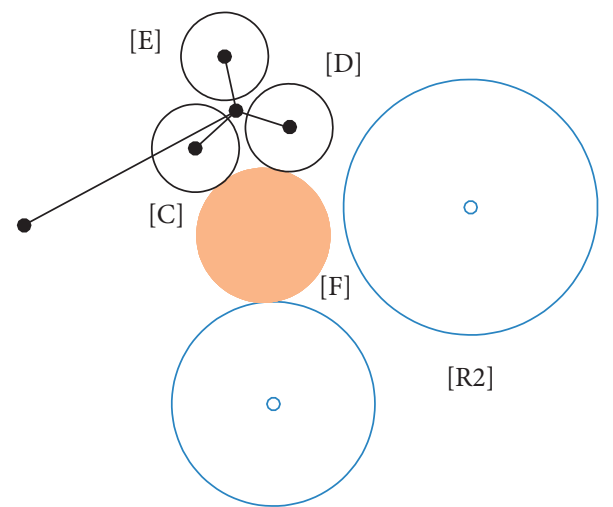

[R1]

(e)

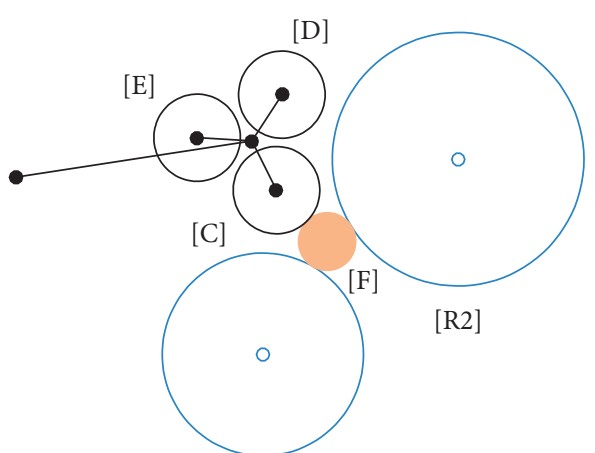

[R1]

(b)

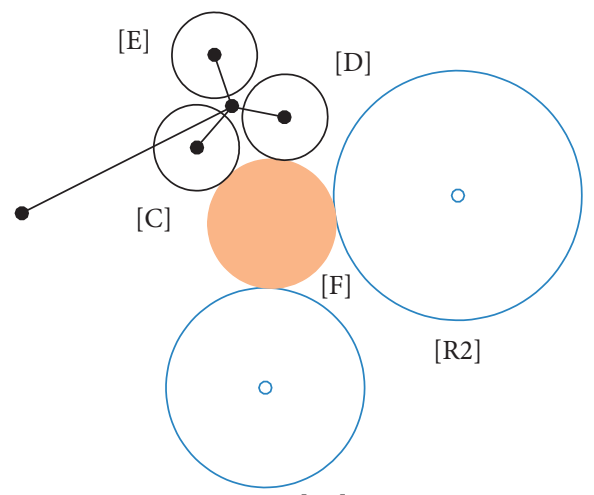

[R1]

(d)

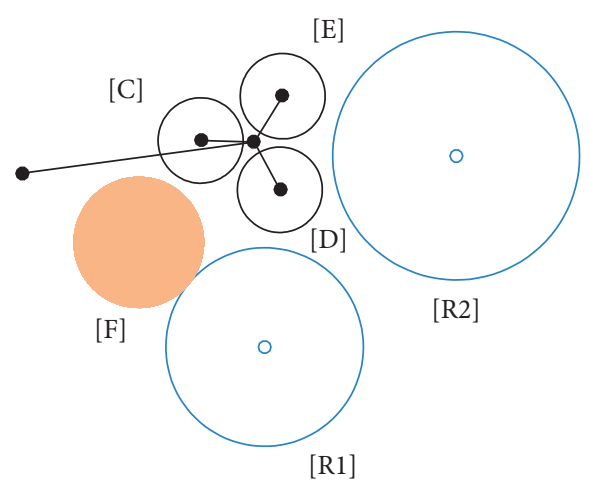

(f)

Figure 4: Characteristic phases of the system motion: (a) Phase I; (b) Phase II; (c) Phase III; (d) Phase IV; (e) Phase V; (f) Phase VI.

$$
\left[\begin{array}{cc}
L_{1}-L_{2} \cos (\Delta \phi) & -L_{2} \sin (\Delta \phi) \\
L_{2} \sin (\Delta \phi) & L_{1}-L_{2} \cos (\Delta \phi)
\end{array}\right]\left[\begin{array}{c}
\cos \left(\phi_{1}\right) \\
\sin \left(\phi_{1}\right)
\end{array}\right]=\left[\begin{array}{c}
d \cos (\phi) \\
d \sin (\phi)
\end{array}\right]
$$

which can be solved to determine the expression of the trajectory of the paper log as a function of its instantaneous radius as

$$
\phi_{1}=\arctan \left(\frac{\left(R_{1}+R_{L}\right) \sin (\phi)-\left(R_{2}+R_{L}\right) \sin (\Delta \phi+\phi)}{\left(R_{1}+R_{L}\right) \cos (\phi)-\left(R_{2}+R_{L}\right) \cos (\Delta \phi+\phi)}\right) \quad \text { if } 2 R_{L}>d-R_{1}-R_{2}
$$




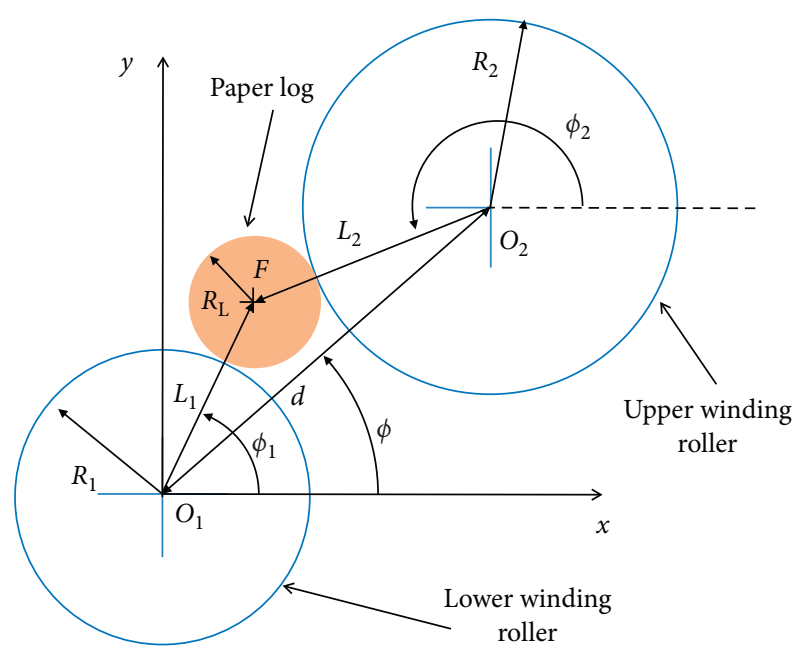

FIgURE 5: Geometric parameters of the system.

The condition in equation (6) is due to the fact that a real solution exists only in the case when the paper log diameter is greater than the distance between the two circles corresponding to the winding rollers.

The Cartesian trajectory of the paper log axis F, in the reference frame $x y$ in Figure 5, is given by

$$
\begin{aligned}
& F_{x}=\left(R_{1}+R_{L}\right) \cos \left(\phi_{1}\right), \\
& F_{y}=\left(R_{1}+R_{L}\right) \sin \left(\phi_{1}\right) .
\end{aligned}
$$

Once the final diameter of the paper log has been reached, the paper log is disengaged from the upper winding roller due to the proper motion of the pressure unit. Until the contact of the paper log with the lower winding roller is assured, the law of motion of the paper log will follow equation (7), in which $R_{L}=R_{L f}$, and the trend of the angle $\phi_{1}$ is selected based on how quickly the product is being ejected. It is worth to note that if it is necessary to temporarily stop the rotation of the pressure unit in the final winding phase, it is sufficient to keep $\phi_{1}$ constant during the desired duration. As an example, Figure 6 shows as a dashed line, the trajectory followed by the axis of the paper log being rolled up.

It is also important to note that a kink is present in the trajectory of motion when the paper log disengages from the upper winding roller. Such kink is due to the different trajectory just before and after said disengagement.

\section{Kinematics of the Pressure Unit}

In this section, the variation of the joint angles in $A$ and $B$ is related to the paper log being rolled up, i.e., to its instantaneous radius and thus to the winding speed according to equations (1a) and (1b).

The required trajectory is designed so that the pressure unit is able to convoy the paper log roll-up and return back to the initial configuration, ready for the next paper log engagement.

The required motion must thus (1) guarantee a smooth trajectory for both the main and secondary arms of the pressure unit, (2) avoid collisions with the other rollers

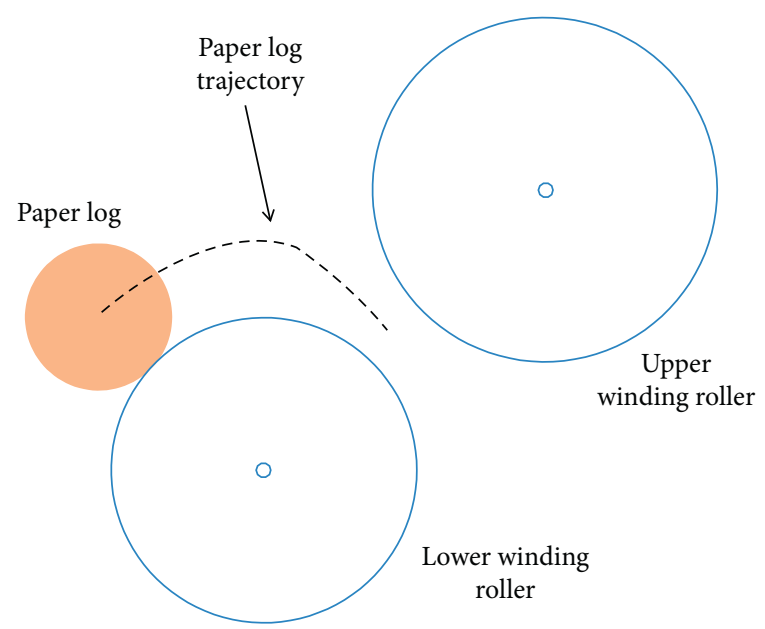

FIgURE 6: Example of a trajectory (dashed line) of the paper log being rolled up.

located in the workspace, and (3) guarantee an optimal orientation of the line of pressure between the pressure roller(s) and the paper log.

In order to determine the kinematics of the pressure unit, i.e., the variation of the joints in $\mathrm{A}$ and $\mathrm{B}$, it is important to identify two cases, according to the condition where one or two pressure rollers are in contact with the paper log. The first case is discussed in Section 4.1 and the second case in Section 4.2 as follows.

4.1. One Pressure Roller in Contact with the Paper Log. The pressure unit and its main parameters for this case are illustrated in Figure 7. In particular, Figure 7(a) shows the overall view and geometry, while Figure 7(b) shows a closeup of the contact between the pressure roller and the paper $\log$.

The main arm of the pressure unit is rotating around the fixed axis $\mathrm{A}$ in Figure 7(a), whose coordinates are $A_{x}$ and $A_{y}$ respect to the frame of reference $x y$. The secondary arms supporting the three pressure rollers are rotating around the axis $\mathrm{B}$, which is at a constant distance $k_{1}$ from axis $\mathrm{A}$. The distance between axis $B$ and the axis of rotation of each of the pressure rollers is constant and labelled as $k_{2}$ in Figure $7(\mathrm{a})$.

The pressure unit has two degrees-of-freedom, and the corresponding generalized coordinates are labelled as the angles $\theta_{1}$ and $\theta_{2}$ in Figure $7(\mathrm{a})$, no matter the mechanical actuation system specifically adopted. The mobility of the system is such that an optimal direction for the line of pressure between the pressure roller and the paper log can be assured. As discussed in Section 3, such direction is perpendicular to the line passing through the centres of rotation of the two winding rollers.

An offset parameter $a$ is conveniently introduced to allow varying the initial location of the pressure roller at first engagement, depending on the type of paper to be rolled up (e.g., thicker and soft; thinner and compact).

Being $\mathrm{C}$ the desired location of the centre of the first engaging pressure roller, as detailed in Figure 7(b), its coordinates are determined as 


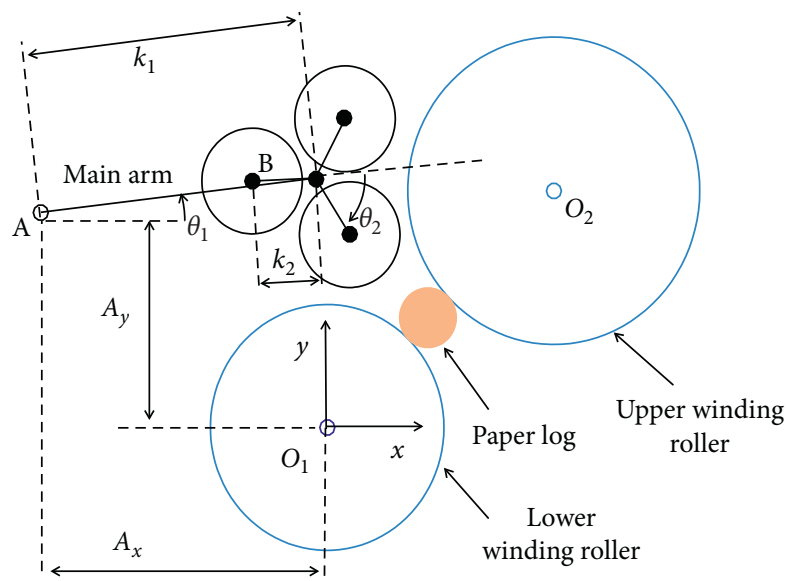

(a)

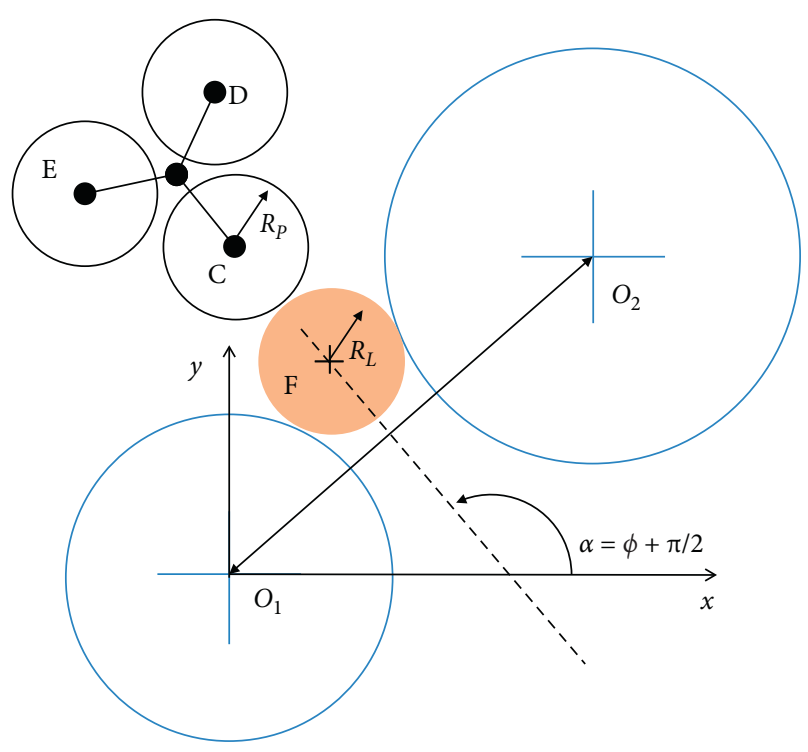

(b)

Figure 7: (a) Main geometry and (b) close-up of the pressure unit in case of one pressure roller in contact with the paper log.

$$
\begin{aligned}
& C_{x}=F_{x}+\left(R_{P}+R_{L}+a\right) \cos \left(\phi+\frac{\pi}{2}\right), \\
& C_{y}=F_{y}+\left(R_{P}+R_{L}+a\right) \sin \left(\phi+\frac{\pi}{2}\right),
\end{aligned}
$$

where $R_{\mathrm{p}}$ is the radius of each pressure roller, and the closure equations of the linkage in the $x y$ reference plane can be thus written as

$$
\begin{aligned}
& A_{x}+k_{1} \cos \theta_{1}+k_{2} \cos \left(\theta_{1}+\theta_{2}\right)=C_{x} . \\
& A_{y}+k_{1} \sin \theta_{1}+k_{2} \sin \left(\theta_{1}+\theta_{2}\right)=C_{y} .
\end{aligned}
$$

Squaring and adding equation (9), the following expression is obtained for the second joint:

$$
\theta_{2}= \pm \arccos \frac{\left(C_{x}-A_{x}\right)^{2}+\left(C_{y}-A_{y}\right)^{2}-k_{1}^{2}-k_{2}^{2}}{2 k_{1} k_{2}} .
$$

As noted before, the sign ambiguity in equation (10) comes from the two different configurations of the linkage. The configuration corresponding to the minus sign is that of physical interest in this specific problem, where the secondary arms rotate clockwise.

Substituting equation (10) into equation (9), the following linear system of equations is determined:

$$
\left[\begin{array}{cc}
k_{1}+k_{2} \cos \left(\theta_{2}\right) & -k_{2} \sin \left(\theta_{2}\right) \\
k_{2} \sin \left(\theta_{2}\right) & k_{1}+k_{2} \cos \left(\theta_{2}\right)
\end{array}\right]\left[\begin{array}{c}
\cos \left(\theta_{1}\right) \\
\sin \left(\theta_{1}\right)
\end{array}\right]=\left[\begin{array}{c}
C_{x}-A_{x} \\
C_{y}-A_{y}
\end{array}\right],
$$

which can be first solved for the unknowns $\cos \left(\theta_{1}\right)$ and $\sin \left(\theta_{1}\right)$ and then solved in terms of the first joint angle yielding

$$
\theta_{1}=\arctan \left(\frac{\left(C_{y}-A_{y}\right)\left(k_{1}+k_{2} \cos \left(\theta_{2}\right)\right)-\left(C_{x}-A_{x}\right) k_{2} \sin \left(\theta_{2}\right)}{\left(C_{x}-A_{x}\right)\left(k_{1}+k_{2} \cos \left(\theta_{2}\right)\right)+\left(C_{y}-A_{y}\right) k_{2} \sin \left(\theta_{2}\right)}\right)
$$

The Cartesian coordinates of point B and the centres D and $\mathrm{E}$ of the pressure rollers in Figure 7(b) can be determined by the following expressions, respectively:

$$
\begin{aligned}
& B_{x}=A_{x}+k_{1} \cos \left(\theta_{1}\right), \\
& B_{y}=A_{y}+k_{1} \sin \left(\theta_{1}\right),
\end{aligned}
$$

$$
\begin{aligned}
& D_{x}=B_{x}+k_{2} \cos \left(\theta_{1}+\theta_{2}+\pi \frac{2}{3}\right), \\
& D_{y}=B_{y}+k_{2} \sin \left(\theta_{1}+\theta_{2}+\pi \frac{2}{3}\right), \\
& E_{x}=B_{x}+k_{2} \cos \left(\theta_{1}+\theta_{2}+\pi \frac{4}{3}\right), \\
& E_{y}=B_{y}+k_{2} \sin \left(\theta_{1}+\theta_{2}+\pi \frac{4}{3}\right) .
\end{aligned}
$$

The following conditions are to be avoided. 


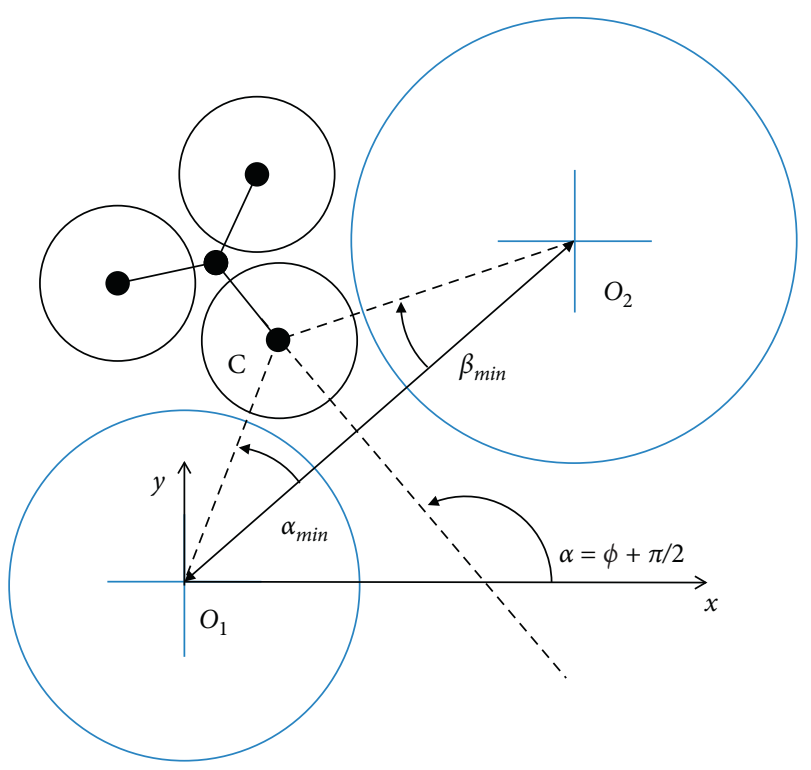

Figure 8: Geometry for the condition of collision between the pressure roller and the winding rollers.

4.1.1. Collision between Pressure Roller [C] and Upper Winding Roller [R2]. When the pressure unit is approaching the upper roller, the following condition is required:

$$
\sqrt{\left(C_{x}-d \cos \phi\right)^{2}+\left(C_{y}-d \sin \phi\right)^{2}}>R_{2}+R_{P} .
$$

Should equation (16) not being satisfied, the pressure unit should hold in place waiting for engagement at the following safety coordinates:

$$
\begin{aligned}
& C_{x}=d \cos (\phi)-\left(R_{P}+R_{2}+\varepsilon_{r}\right) \cos \left(\phi-\beta_{\text {min }}\right), \\
& C_{y}=d \sin (\phi)-\left(R_{P}+R_{2}+\varepsilon_{r}\right) \sin \left(\phi-\beta_{\text {min }}\right),
\end{aligned}
$$

which replaces equation (8), where $\varepsilon_{r}$ is a safety parameter providing an adequate distance tolerance among the rollers, and

$$
\beta_{\text {min }}=\arccos \left(\frac{\left(d+R_{2}-R_{1}\right) / 2}{\varepsilon_{r}+R_{2}+R_{P}}\right),
$$

which is depicted in Figure 8.

4.1.2. Collision between Pressure Roller [C] and Lower Winding Roller [R1]. When the pressure unit is approaching the lower roller, the following condition is required:

$$
\sqrt{C_{x}^{2}+C_{y}^{2}}>R_{1}+R_{P}
$$

Should equation (19) not being satisfied, the pressure unit should hold in place waiting for engagement at the following safety coordinates:

$$
\begin{aligned}
& C_{x}=\left(R_{P}+R_{1}+\varepsilon_{r}\right) \cos \left(\phi+\alpha_{\text {min }}\right), \\
& C_{y}=\left(R_{P}+R_{1}+\varepsilon_{r}\right) \sin \left(\phi+\alpha_{\text {min }}\right),
\end{aligned}
$$

which again replaces equation (8), where

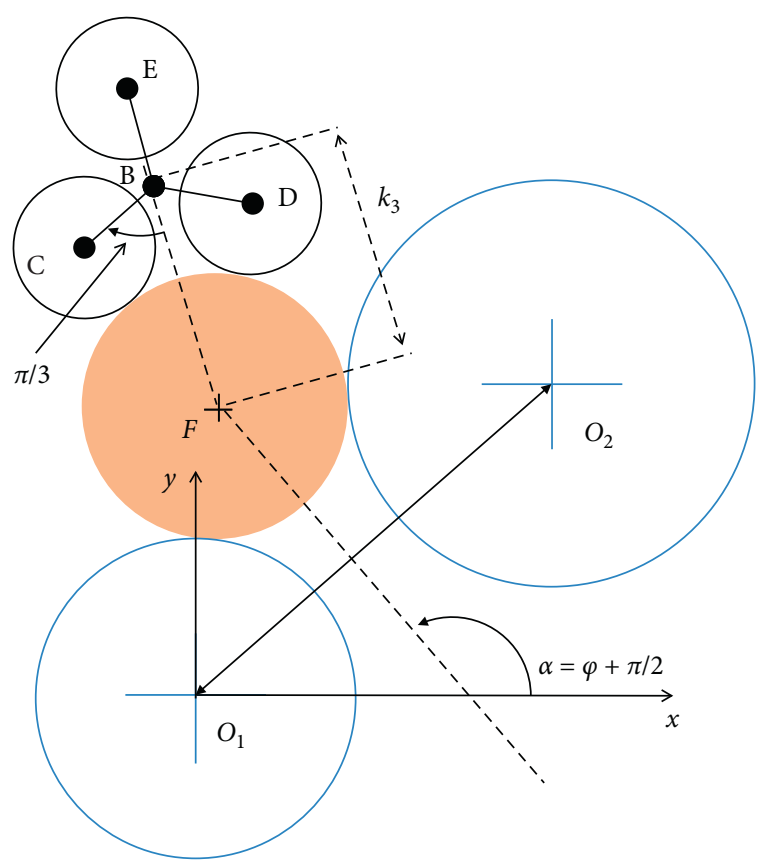

FIGURE 9: Configuration where the paper log is in contact with two pressure rollers.

$$
\alpha_{\min }=\arccos \left(\frac{\left(d+R_{1}-R_{2}\right) / 2}{\varepsilon_{r}+R_{1}+R_{P}}\right),
$$

which is depicted in Figure 8.

4.1.3. Collision between Pressure Roller [D] and Upper Winding Roller [R2]. Since the pressure unit is rotating clockwise, when the pressure roller [D] is approaching the engagement location, the following condition should be satisfied:

$$
\sqrt{\left(D_{x}-d \cos \phi\right)^{2}+\left(D_{y}-d \sin \phi\right)^{2}}>R_{2}+R_{P}
$$

In this case, should equation (22) not being satisfied, the pressure unit cannot guarantee the optimal condition for the line of pressure between the pressure roller and the paper $\log$.

4.2. Two Pressure Rollers in Contact with the Paper Log. The equations in Section 4.1 are valid until only the pressure roller $[\mathrm{C}]$ is in contact with the paper log being rolled up. It is worth noting that the pressure roller [C] remains tangent to the paper log at the point of contact between the paper log circle and the line orthogonal to the centres of the winding rollers, assuring the optimal line of pressure.

Due to the increase in the paper log diameter, the pressure unit rotates and the pressure roller $[\mathrm{D}]$ approaches the paper log. The contact between the pressure roller [D] and the paper log is achieved when

$$
\sqrt{\left(D_{x}-F_{x}\right)^{2}+\left(D_{y}-F_{y}\right)^{2}}<R_{L}+R_{P}+a \text {. }
$$


In this case, the paper log is constrained by four rollers, two winding rollers and two pressure rollers. This is illustrated in Figure 9, and the motion of the pressure unit needs to be changed to accomplish such motion. This is the reason for the kink mentioned earlier in Section 3 with reference to Figure 6.

To assure the best possible containment for the paper log being rolled up, the configuration of the pressure unit is selected such as the pressure rollers [C] and [D] in Figure 9 are located symmetrically respect to the line passing through the centre of the paper log and point $\mathrm{B}$. The configuration of the pressure unit is then determined through the Carnot theorem applied to the triangle FCB, in Figure 9, giving

$$
\left(R_{P}+R_{L}\right)^{2}=k_{2}^{2}+k_{3}^{2}-2 k_{2} k_{3} \cos \left(\frac{\pi}{3}\right),
$$

which can be rewritten as a quadratic equation in terms of $k_{3}$, representing the varying distance between point $B$ and the centre of the paper log, $F$, as

$$
k_{3}^{2}+k_{3}\left(-2 k_{2} \cos \left(\frac{\pi}{3}\right)\right)+\left[k_{2}^{2}-\left(R_{P}+R_{L}\right)^{2}\right]=0 .
$$

Equation (25) can be thus solved to give

$$
k_{3}=k_{2} \cos \left(\frac{\pi}{3}\right) \pm \frac{1}{2} \sqrt{4 k_{2}^{2} \cos \left(\frac{\pi}{3}\right)^{2}-4\left[k_{2}^{2}-\left(R_{P}+R_{L}\right)^{2}\right]}+a \text {. }
$$

The closure equations of the linkage are then determined as

$$
\begin{aligned}
& A_{x}+k_{1} \cos \theta_{1}+k_{3} \cos \left(\theta_{1}+\theta_{2}+\frac{\pi}{3}\right)=F_{x}, \\
& A_{y}+k_{1} \sin \theta_{1}+k_{3} \sin \left(\theta_{1}+\theta_{2}+\frac{\pi}{3}\right)=F_{y} .
\end{aligned}
$$

As detailed in Section 4.1, the new expression of the second joint angle is determined as

$$
\theta_{2}= \pm \arccos \frac{\left(F_{x}-A_{x}\right)^{2}+\left(F_{y}-A_{y}\right)^{2}-k_{1}^{2}-k_{3}^{2}}{2 k_{1} k_{3}}-\frac{\pi}{3}
$$

where the configuration of interest is that corresponding to the minus sign.

Substituting equation (28) into equations (27), the following system of equations is obtained in terms of the unknowns $\cos \left(\theta_{1}\right)$ and $\sin \left(\theta_{1}\right)$ :

$$
\left[\begin{array}{cc}
k_{1}+k_{3} \cos \left(\theta_{2}+\frac{\pi}{3}\right) & -k_{3} \sin \left(\theta_{2}+\frac{\pi}{3}\right) \\
k_{3} \sin \left(\theta_{2}+\frac{\pi}{3}\right) & k_{1}+k_{3} \cos \left(\theta_{2}+\frac{\pi}{3}\right)
\end{array}\right]\left[\begin{array}{c}
\cos \left(\theta_{1}\right) \\
\sin \left(\theta_{1}\right)
\end{array}\right]=\left[\begin{array}{c}
F_{x}-A_{x} \\
F_{y}-A_{y}
\end{array}\right],
$$

and it can be solved for the first joint angle as

$$
\theta_{1}=\arctan \left(\frac{\left(A_{x}-F_{x}\right) k_{3} \cos \left((\pi / 6)-\theta_{2}\right)-\left(A_{y}-F_{y}\right)\left(k_{1}+k_{3} \sin \left((\pi / 6)-\theta_{2}\right)\right)}{-\left(A_{y}-F_{y}\right) k_{3} \cos \left((\pi / 6)-\theta_{2}\right)-\left(A_{x}-F_{x}\right)\left(k_{1}+k_{3} \sin \left((\pi / 6)-\theta_{2}\right)\right)}\right)
$$

4.3. Early Engagement of the Second Pressure Roller. In the case in which it is desired to anticipate the engagement of the second pressure roller with the paper log (e.g., to prevent paper log instability), equation (8) is replaced by

$$
\begin{aligned}
& C_{x}=F_{x}+\left(R_{P}+R_{L}+a\right) \cos \left(\phi+\frac{\pi}{2}+\gamma\right), \\
& C_{y}=F_{y}+\left(R_{P}+R_{L}+a\right) \sin \left(\phi+\frac{\pi}{2}+\gamma\right),
\end{aligned}
$$

where $\gamma$ is an angular parameter varying from zero to a given final value, which depends on the desired conditions for engagement. In particular, a value for the paper log radius, $R_{L a}$, at which the early engagement begins, is to be defined, as well as a value for the paper log radius, $R_{L b}$, at which the final engagement of the second pressure roller with the paper log occurs. It is worth noting that, during the phase from $R_{L a}$ to $R_{L b}$, it is not possible to assure the optimal condition for the line of pressure between the pressure roller and the paper log, as described in Section 4.1.

Once the values for $R_{L a}$ and $R_{L b}$ have been specified, the value of $\gamma$ varies from $\gamma_{a}=0$, corresponding to the paper log radius $R_{L a}$, to a final value of 
TABLE 1: Geometric parameters used for simulations.

\begin{tabular}{lccc}
\hline Parameter & Unit & Case 1 & Case 2 \\
\hline$D$ & $(\mathrm{~mm})$ & & 253.5 \\
$\Phi$ & $(\mathrm{rad})$ & & $\pi / 4$ \\
$R_{1}$ & $(\mathrm{~mm})$ & & 97.5 \\
$R_{2}$ & $(\mathrm{~mm})$ & 48.8 & 110 \\
$R_{L i}$ & $(\mathrm{~mm})$ & - & 23.2 \\
$R_{L f}$ & $(\mathrm{~mm})$ & - & 72.0 \\
$R_{L a}$ & $(\mathrm{~mm})$ & & - \\
$R_{L b}$ & $(\mathrm{~mm})$ & & -220.75 \\
$A_{x}$ & $(\mathrm{~mm})$ & & 160.75 \\
$A_{y}$ & $(\mathrm{~mm})$ & & 218 \\
$k_{1}$ & $(\mathrm{~mm})$ & $(\mathrm{mm})$ & 49.07 \\
$k_{2}$ & $(\mathrm{~mm})$ & 32.5 \\
$R_{P}$ & $(\mathrm{~mm})$ & 10 \\
$\varepsilon_{r}$ & & & 72.0 \\
\hline
\end{tabular}

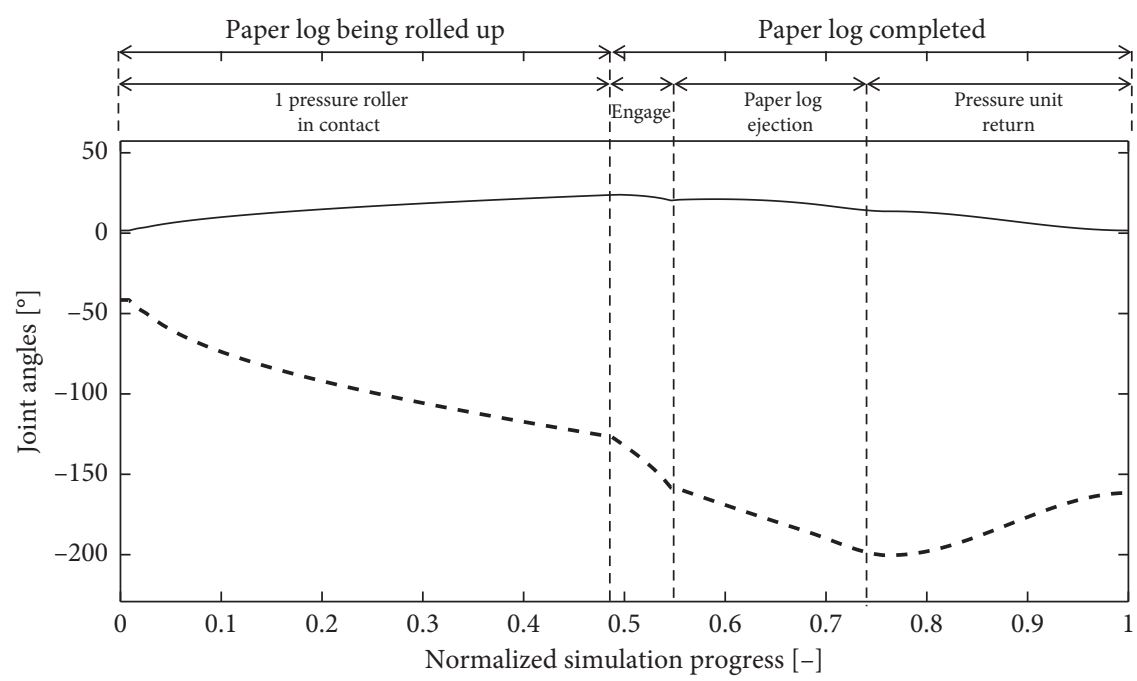

$-\theta_{1}$

Figure 10: Simulation results for Case 1.

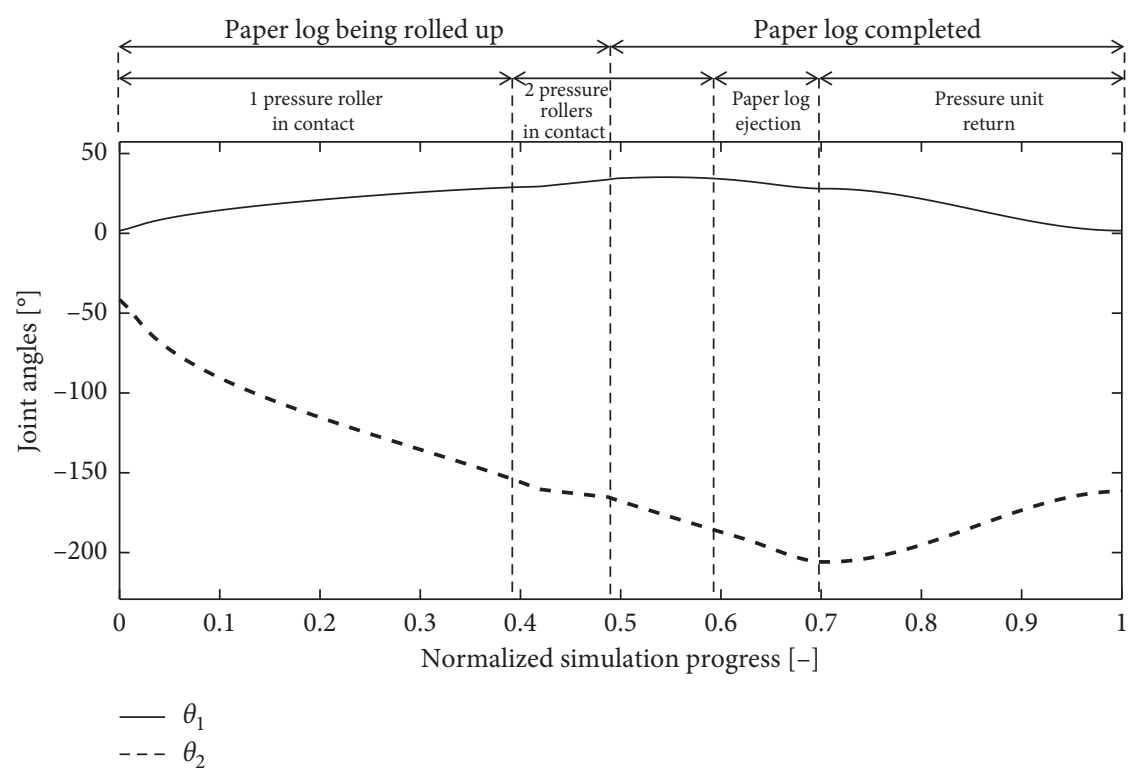

FIGURE 11: Simulation results for Case 2. 


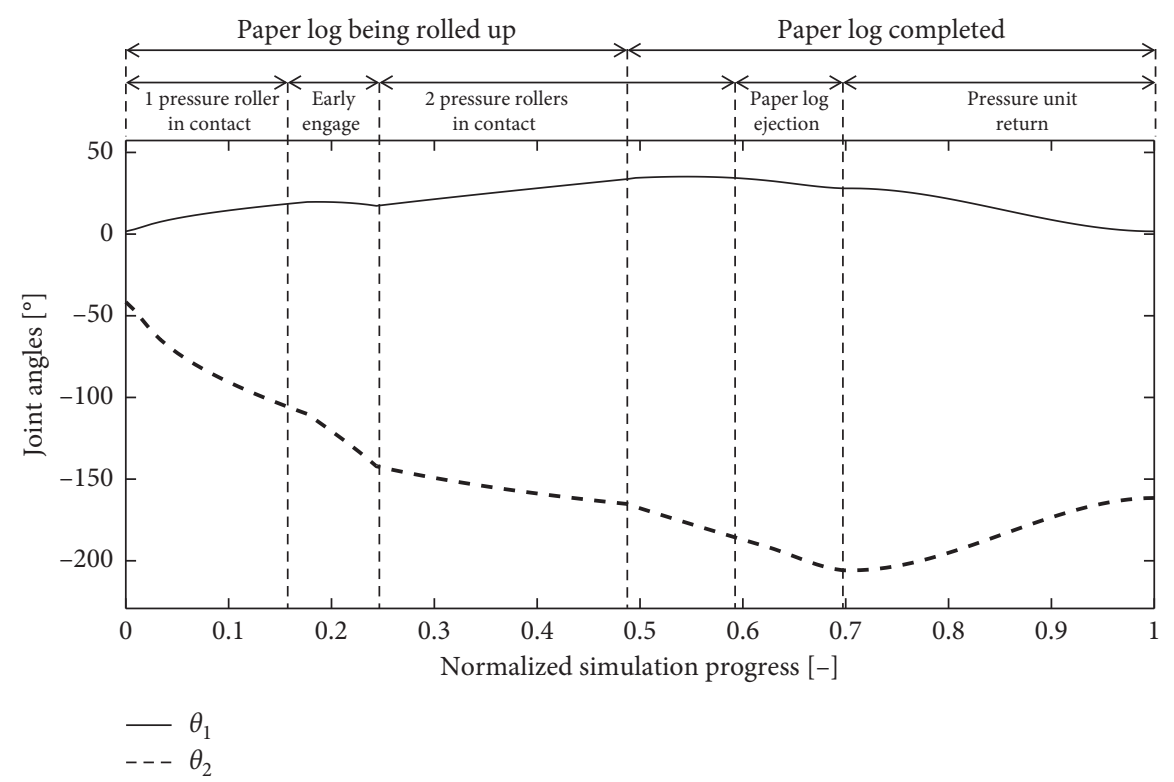

FIgURE 12: Simulation results for Case 3.

$$
\gamma_{b}=\arccos \left(\frac{\left(C_{x b}-F_{x b}\right) \cos (\phi+(\pi / 2))+\left(C_{y b}-F_{y b}\right) \sin (\phi+(\pi / 2))}{\sqrt{\left(C_{x b}-F_{x a}\right)^{2}+\left(C_{y b}-F_{y a}\right)^{2}}}\right),
$$

which corresponds to the paper $\log$ radius $R_{L b}$, where $F_{x a}$ and $F_{x b}$ are the coordinates of the position of the paper log axis at radius $R_{L b}$, the coordinates $C_{x b}$ and $C_{y b}$ are determined on the base of the corresponding configuration achieved by the system with four rollers in contact, as detailed in Section 4.2.

4.4. Kinematics of the Pressure Unit after Log Ejection. The optimal condition for ejection of the paper log is achieved when the angle of the tangent to the pressure roller $[\mathrm{C}]$ and the paper log is less than that of the tangent to the lower winding roller and the paper log so that the following relation is satisfied:

$$
\arctan \left(\frac{C_{y}-F_{y}}{C_{x}-F_{x}}\right) \leq \phi_{1} .
$$

In this case, the paper log is smoothly released without the need to disengage the pressure unit, which is then free to return back to its initial position for the next engagement. Such a motion can be defined by a polynomial blending trajectory, which assures the required conditions for continuity and smoothness. For each joint angle $\theta_{1}$ and $\theta_{2}$, the initial boundary conditions are given by equations (28) and (30) at the time of ejection. The final boundary conditions have to guarantee that the pressure unit returns back to its position with zero final velocity (at least) and with pressure roller [D] replacing [C] -this is achieved by an offset for $\theta_{2}$ equal to $-120^{\circ}$ respect to the previous initial configuration.

\section{Application}

Applications are provided in this section to illustrate the device motion and to provide validation to the algorithm presented in the previous sections.

Three different simulations are performed, according to the geometric parameters listed in Table 1. For the three cases, the difference in the parameters is related to the final diameter of the paper log, which is smaller in Case 1 than in Case 2, and the early engagement of the second pressure roller, which is absent in Case 2 and present in Case 3.

Results of the simulations are reported in Figures 10-12 for the three cases, respectively. They are presented in terms of the joint angles of the pressure unit as functions of a nondimensional simulation time and are derived based on the inverse kinematic algorithm presented in the sections above. The various kinks in the joint trajectories denote a change from one contact phase to a different one. An additional kink in Figure 12 is due to the early engagement of the second pressure roller.

The results of the inverse kinematics shown in Figures 10-12 are verified by solving the direct kinematics of the system and combining it with the imposed trajectory of the paper log during roll-up. The results of such validation are presented as animation sequences in the video S1, S2, and S3, respectively, provided in the Supplementary Material (Available here). It can be seen how the pressure unit follows the paper log during the different phases of the designed trajectories. 


\section{Conclusions}

In this paper, a detailed kinematic analysis of a novel mechanical pressure unit integrated in a rewinding paper machine is presented. The system is based on a recent patent application, and it allows the creation of paper logs with different diameters by assuring adequate constraints during winding. The novelty of the system relies on the use of a pressure unit with three pressure rollers, respect to standard configurations with one roller only. The proposed system has two degrees-of-freedom, which need to be synchronized in order to adequately restrain and copy the paper roll motion. This allowed to increase the overall winding speed reducing machine downtime and motivated the introduced complexity respect to classical one degree-of-freedom pressure units. The trajectories of the two joint angles of the pressure unit are determined through an inverse kinematic analysis, and the conditions for collision are identified and taken into account in the algorithm. Numerical applications are presented which illustrate the motion of the pressure unit along with the paper log during formation. Some of the different possible scenarios are implemented. The algorithm presented in the paper is validated through the direct kinematics of the system.

\section{Abbreviations}

$t: \quad$ Time

$V: \quad$ Winding speed

s: $\quad$ Paper thickness

$R_{L}$ : Radius of the paper $\log$ at time $t$

$R_{L i}: \quad$ Initial radius of the paper $\log$

$R_{L i}: \quad$ Final radius of the paper log

$L: \quad$ Paper wrapped on each paper log

$R_{1}$ : $\quad$ Radius of the lower winding roller

$R_{2}$ : $\quad$ Radius of the upper winding roller

$R_{P}: \quad$ Radius of the pressure rollers

$d$ : Constant distance between the lower and upper winding roller

$\phi$ : Constant angle between horizontal axis and the direction of $d$

$L_{1}$ : $\quad$ Variable distance between the lower winding roller and the paper log

$\phi_{1}$ : Variable angle between horizontal axis and the direction of $L_{1}$

$L_{2}$ : Variable distance between the upper winding roller and the paper log

$\phi_{2}$ : Variable angle between horizontal axis and the direction of $L_{2}$

$\Delta \phi: \quad$ Difference between $\phi_{1}$ and $\phi_{2}$

$P_{x}, \quad$ Coordinate $x$ and $y$ of a generic point $P$

$P_{y}:$

$\theta_{1}: \quad$ Joint angle of the main arm

$\theta_{2}$ : Joint angle of the pressure unit

$k_{1}$ : $\quad$ Constant length of the main arm

$k_{2}$ : Constant length of second arms of the pressure unit

$k_{3}$ : Variable distance between the centre of the paper log and secondary pivot joint of the pressure unit

$a$ : Offset of the pressure roller respect to the paper log at first engagement $\varepsilon_{r}: \quad$ Tolerance distance among the rollers

$\gamma$ : Angular parameter defining early engagement

$\gamma_{a}$ : Angular parameter where early engagement begins

$\gamma_{b}$ : Angular parameter where early engagement ends

$R_{L a}: \quad$ Radius of the paper log where early engagement begins

$R_{L b}$ : Radius of the paper log where early engagement ends.

\section{Data Availability}

The data used to support this study are provided within the article.

\section{Disclosure}

This research work was performed under a research agreement between the Department of Mechanical, Energy and Management Engineering of the University of Calabria and the private company United Converting Tissue Srl.

\section{Conflicts of Interest}

The authors declare that they have no conflicts of interest.

\section{Acknowledgments}

The research work related to this article was partially funded by the private company United Converting Tissue Srl, under a research agreement with the Department of Mechanical, Energy, and Management Engineering of the University of Calabria. L. Lupi and G. Lupi would like to acknowledge Mr. Luca Salotti and Mr. Christian Moscardini for the development of the system software.

\section{Supplementary Materials}

Supplementary Materials are provided for this paper as follows: S0: animation comparing the proposed system to a traditional one. S1: animation illustrating application Case 1. S2: animation illustrating application Case 2. S3: animation illustrating application Case 3. (Supplementary Materials)

\section{References}

[1] T.-L. Liu, "Kinematic analysis on winding and unwinding process in tissue paper machine and its application," China Pulp and Paper, vol. 36, no. 1, pp. 52-56, 2017.

[2] D. R. Roisum, "Paper winding," in Lignocellulosic Fibers and Wood Handbook: Renewable Materials for Today's Environment, pp. 463-479, Hoboken, NJ, USA, 2016.

[3] W.-M. Ma and X. Chen, "Design of constant tension control system for paper rewinding machine based on IMC," China Pulp and Paper, vol. 37, no. 12, pp. 56-62, 2018.

[4] F.-Y. Ye and M.-Y. Hu, "Study on the control of paper rewinding of a modern tissue machine," China Pulp and Paper, vol. 37, no. 12, pp. 46-50, 2018.

[5] S.-H. Chen, "Automatic control system and its application in paper rewinder," China Pulp and Paper, vol. 37, no. 1, pp. 42-45, 2018.

[6] R. Carrasco and M. A. Valenzuela, "Tension control of a twodrum winder using paper tension estimation," IEEE 
Transactions on Industry Applications, vol. 42, no. 2, pp. 618-628, 2006.

[7] B. I. Jeftenić and M. Z. Bebić, "Realization of rewinder with a reduced number of sensors," IEEE Transactions on Industrial Electronics, vol. 57, no. 8, pp. 2797-2806, 2010.

[8] M. A. Abd-Elraouf, H. A. Ashour, and A. El-Shenawy, "Performance improvement of automatic tension control in multi coordinate drive systems for industrial paper winder," in Proceedings of the 2017 19th International Middle-East Power Systems Conference, pp. 1163-1169, Cairo, Egypt, December 2017.

[9] W.-X. Gao and Y.-H. Guo, "The application of accessory S00 of SIEMENS in self-adjusting reel diameter calculation," China Pulp and Paper, vol. 30, no. 8, pp. 52-54, 2011.

[10] Y.-P. Guo and W.-B. Yang, "Pressure control principle and scheme of rewinder's platen," China Pulp and Paper, vol. 29, no. 7, pp. 56-58, 2010.

[11] B. Wick and P. Berberian, "Aligning rolls in a paper machine winder," Paper360, vol. 7, no. 2, pp. 30-32, 2012.

[12] G. Lupi, A. Torri, and G. Giometti, Rewinding Machine, WO2018037272 (A1), 2018.

[13] G. Gatti and G. Danieli, "A practical approach to compensate for geometric errors in measuring arms: application to a sixdegree-of-freedom kinematic structure," Measurement Science and Technology, vol. 19, p. 015107, 2008.

[14] J. Liu, Y. Xu, and G. Pan, "A combined acoustic and dynamic model of a defective ball bearing," Journal of Sound and Vibration, vol. 501, p. 116029, 2021. 